\title{
Influence of centrality definition and detector efficiency on the net-proton kurtosis
}

\author{
Sukanya Sombun ${ }^{1}$, Jan Steinheimer ${ }^{2}$, Christoph Herold ${ }^{1}$, Ayut Limphirat ${ }^{1}$, \\ Yupeng Yan $^{1}$, and Marcus Bleicher ${ }^{2,3,4,5}$ \\ 1 School of Physics and Center of Excellence in High Energy Physics \& Astrophysics, \\ Suranaree University of Technology, Nakhon Ratchasima 30000, Thailand \\ 2 Frankfurt Institute for Advanced Studies, Ruth-Moufang-Str. 1, 60438 Frankfurt \\ am Main, Germany \\ 3 Institut für Theoretische Physik, Goethe Universität Frankfurt, \\ Max-von-Laue-Strasse 1, D-60438 Frankfurt am Main, Germany \\ ${ }^{4}$ GSI Helmholtzzentrum für Schwerionenforschung GmbH, Planckstr. 1, 64291 \\ Darmstadt, Germany \\ 5 John von Neumann-Institut für Computing, Forschungzentrum Jülich,52425 Jlich, \\ Germany \\ sukanya_joy33@hotmail.com
}

\begin{abstract}
We study the influence of the centrality definition and detector efficiency on the net-proton kurtosis for minimum bias $\mathrm{Au}+\mathrm{Au}$ collisions at a beam energy of $\sqrt{s_{\mathrm{NN}}}=7.7 \mathrm{GeV}$ by using the UrQMD model. We find that different ways of defining the centrality lead to different cumulant ratios. Moreover, we demonstrate that the kurtosis is suppressed for central collisions when a wider transverse momentum acceptance is used. Finally, the influence of a detector efficiency on the measured cumulant ratios is estimated.
\end{abstract}

Keywords: Centrality definition $\cdot$ Cumulant ratios $\cdot$ Net-proton number fluctuations.

\section{Introduction}

One aim of heavy ion collisions is to study the phase structure of the strong interaction or quantum chromodynamics (QCD) which has been widely investigated both in experiment and theory. QCD based models predict a first-order phase transition at large $\mu_{B}$ which ends in a QCD critical point $(\mathrm{CP})$ [1]. The QCD phase structure can be disclosed from the study of event-by-event fluctuations of conserved quantities which can be defined in the form of cumulants. Especially cumulants of sthe net-proton number are predicted to be sensitive to the presence of a QCD phase transition 223] and CP [456677. Higher order cumulants are particularly sensitive to a divergent correlation length close to the $\mathrm{CP}$. The higher order susceptibilities of baryon number, electric charge and strangeness have been calculated theoretically in 88|9|10]11|12]13]14|15]16]. Experimentally, event-by-event fluctuations of higher order cumulants of net-proton, net-pion 
and net-kaon number were measured by RHIC [17|18|19|20|21|22] and LHC $23 \mid 24$ 25. Hereby, a non-monotonic behaviour of higher order cumulant ratios as a function of beam energy might disclose critical behaviour. Although critical fluctuations have been widely studied, there are still difficulties in understanding the interplay of different effects and their impact on the measured observables. Some uncertainties that we are going to address in this paper using the UrQMD model are the centrality determination, the importance of volume fluctuations, the transverse momentum $\left(p_{T}\right)$ acceptance, and efficiency corrections.

\subsection{The UrQMD model}

For the present study, we use the Ultrarelativistic Quantum Molecular Dynamics (UrQMD) model to study cumulant ratios of the net-proton number distribution. The UrQMD model is a microscopic transport model which is able to simulate $\mathrm{p}+\mathrm{p}, \mathrm{p}+\mathrm{A}$ and $\mathrm{A}+\mathrm{A}$ collisions. It is based on the covariant propagation of constituent quarks and anti-quarks accompanied by mesonic and baryonic degrees of freedom, binary elastic and inelastic scattering of hadrons, resonance excitations as well as string dynamics and strangeness exchange reactions $26 / 27 / 28$. The elementary cross-sections are interpreted geometrically and are taken from experimental data 29$]$.

\section{Method}

\subsection{Calculation of cumulants}

The fluctuations of the net-proton number distribution can be characterized by the corresponding cumulants. These are calculated as

$$
\begin{aligned}
& C_{1}=M=\langle\mathrm{N}\rangle \\
& C_{2}=\sigma^{2}=\left\langle(\delta \mathrm{N})^{2}\right\rangle \\
& C_{3}=S \sigma^{3}=\left\langle(\delta \mathrm{N})^{3}\right\rangle \\
& C_{4}=\kappa \sigma^{4}=\left\langle(\delta \mathrm{N})^{4}\right\rangle-3\left\langle(\delta \mathrm{N})^{2}\right\rangle^{2}
\end{aligned}
$$

where $(\delta \mathrm{N})=\mathrm{N}-\langle\mathrm{N}\rangle$, and $\langle\mathrm{N}\rangle$ is the event-averaged value of the net-proton number N. We consider the following ratios of these cumulants,

$$
\begin{aligned}
& \frac{C_{2}}{C_{1}}=\frac{\left\langle\delta N^{2}\right\rangle}{\langle N\rangle}=\frac{\sigma^{2}}{M} \\
& \frac{C_{3}}{C_{2}}=\frac{\left\langle\delta N^{3}\right\rangle}{\left\langle\delta N^{2}\right\rangle}=S \sigma \\
& \frac{C_{4}}{C_{2}}=\frac{\left\langle\delta N^{4}\right\rangle}{\left\langle\delta N^{2}\right\rangle}-3\left\langle\delta N^{2}\right\rangle=\kappa \sigma^{2}
\end{aligned}
$$

with combinations of mean $(\mathrm{M})$, variance $\left(\sigma^{2}\right)$, skewness $(S)$ and kurtosis $(\kappa)$ of measured event-by-event fluctuations. For the statistical error calculations, we 
use the the delta theorem [30] which states that the error of cumulant ratios is proportional to a certain power of the standard deviation as:

$$
\operatorname{error}\left(\frac{C_{r}}{C_{2}}\right) \propto \frac{\sigma^{r-2}}{\sqrt{n}} .
$$

Here, $r$ denotes the order of the cumulant, and $n$ the number of events.

\subsection{Centrality definition}

In heavy-ion experiments, there is no unique definition of centrality. As the impact parameter $(b)$ is experimentally not accessible, observables like the number of participants $\left(N_{\text {part }}\right)$ or the number of charged particles $\left(N_{\text {charge }}\right)$ are used. These can be related to the impact parameter e.g. by a Glauber model. Therefore, the centrality is practically determined by particle multiplicities. In our work, the centrality will be defined by $N_{\text {part }}, N_{\text {charge }}$ or the number of participants in the projectile, $N_{\text {part-projectile }}$. Calculation of cumulants as a function of $N_{\text {part }}$ and $N_{\text {charge }}$ is believed to minimize volume fluctuations which can have an influence on the extracted ratio of cumulants. We study the dependence of the net-proton number on the different centrality definitions. For this, we simulate $\mathrm{Au}+\mathrm{Au}$ collision at a beam energy of $\sqrt{s_{\mathrm{NN}}}=7.7 \mathrm{GeV}$ with impact parameters in the range $0 \leq b \leq 15 \mathrm{fm}$. We define the following quantities,

- $N_{\text {charge }}$ : The number of all charged particles with $|\eta| \leq 1$ and $0.15<p_{T}<$ $2.0 \mathrm{GeV}$ minus the number of protons and anti-protons in this specific acceptance range.

- $N_{\text {part }}$ : 394 minus all spectator protons and neutrons defined by $|y|>1.5$ and $p_{T}<0.3 \mathrm{GeV}$.

- $N_{\text {part-projectile }}: 197$ minus all projectile spectator protons and neutrons defined by $y>1.5$ and $p_{T}<0.3 \mathrm{GeV}$.

We determine the distribution of these three different quantities as shown in figure 1. The three different methods give different distributions. We find that the participant distribution of $N_{\text {part }}$ shows a sharp cutoff at the maximum number of participants, whereas the distribution of $N_{\text {charge }}$ shows a much smoother drop. The centrality is classified into 10 centrality bins of $0-10 \%, 10-20 \%, 20-30 \%, 30$ $40 \%, 40-50 \%, 50-60 \%, 60-70 \%, 70-80 \%, 80-90 \%$ and $90-100 \%$. We calculate the cumulants $C_{n}$ for a fixed $N_{\text {part }}\left(N_{\text {charge }}, N_{\text {part-projectile }}\right)$ and then average those cumulants over all $N_{\text {part }}$ in a given centrality bin. The ratios of cumulants are then determined as ratios of averages.

\section{Results}

\subsection{Dependence on centrality definition}

We first assume that the efficiency of the detector and its acceptance are perfect $(100 \%)$ for all particles. Figure 2 shows the result of the net-proton number 


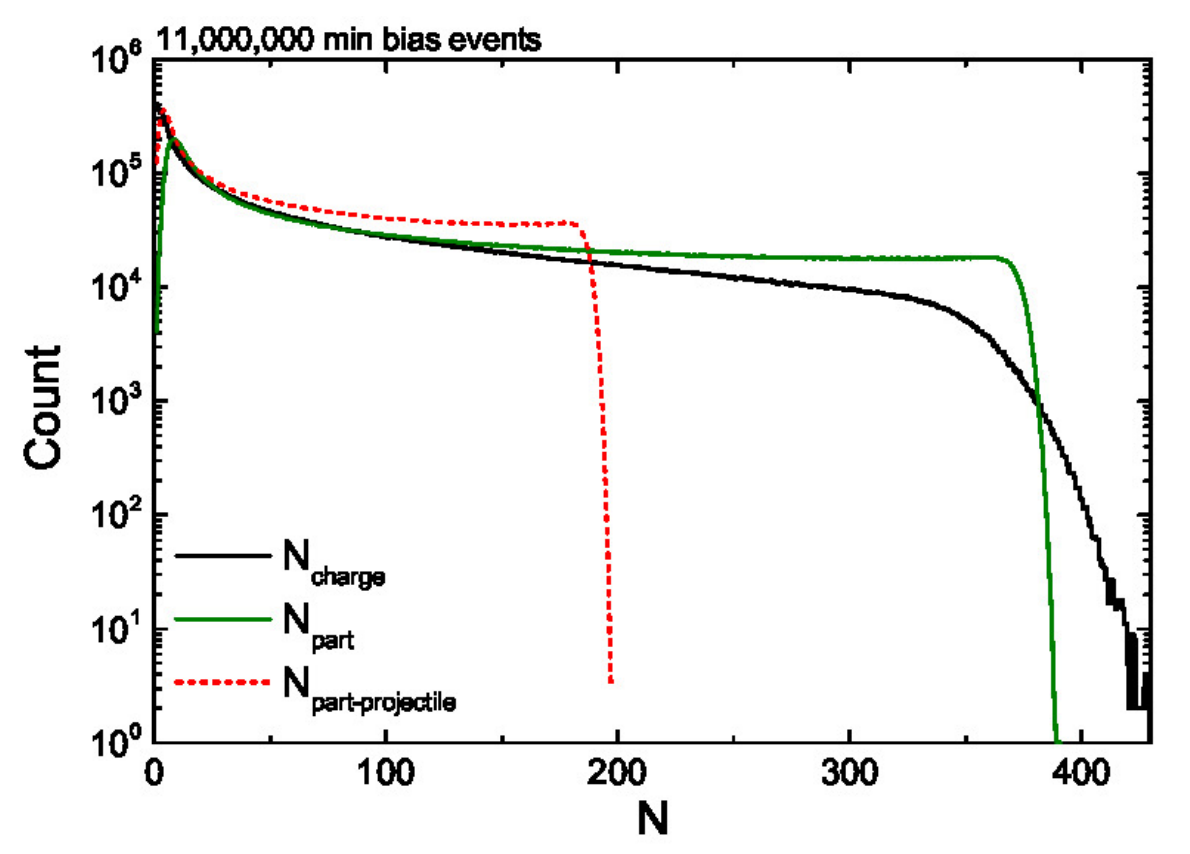

Fig. 1. [Color online] Distributions of $N_{\text {charge }}, N_{\text {part }}$ and $N_{\text {part-projectile }}$ in $\mathrm{Au}+\mathrm{Au}$ collision at a beam energy of $\sqrt{s_{\mathrm{NN}}}=7.7 \mathrm{GeV}$ with impact parameter $0 \leq b \leq 15 \mathrm{fm}$. Figure from [31.

kurtosis as a function of centrality for the three centrality definitions, at midrapidity $|y|<0.5$ and within transverse momentum $0.4<p_{T}<0.8 \mathrm{GeV}$. For the most central collisions, we find that the value of the kurtosis does not depend on the centrality definition. On the other hand, for mid-central collision, the difference between the values of $\kappa \sigma^{2}$ becomes larger. Moreover, the centrality defined by $N_{\text {charge }}$ yields only a mild dependence of the kurtosis on the centrality because it not subject to large volume fluctuations. Thus, we use $N_{\text {charge }}$ in what follows to define the centrality to investigate other effects.

\subsection{Effect of transverse momentum range}

We now study the kurtosis of the net-proton number as a function of centrality which is defined by $N_{\text {charge }}$ for two different transverse momentum ranges, namely $\left(0.4<p_{T}<0.8 \mathrm{GeV}\right)$ and $\left(0.4<p_{T}<2.0 \mathrm{GeV}\right)$. The result is shown in figure 3 . It can be seen that for the most central collisions, the value of the kurtosis is strongly suppressed for the larger acceptance range due to baryon number conservation. At mid-central collisions, the value of the kurtosis is larger for the wider transverse momentum range which indicates that volume fluctuations affect the extracted kurtosis. 


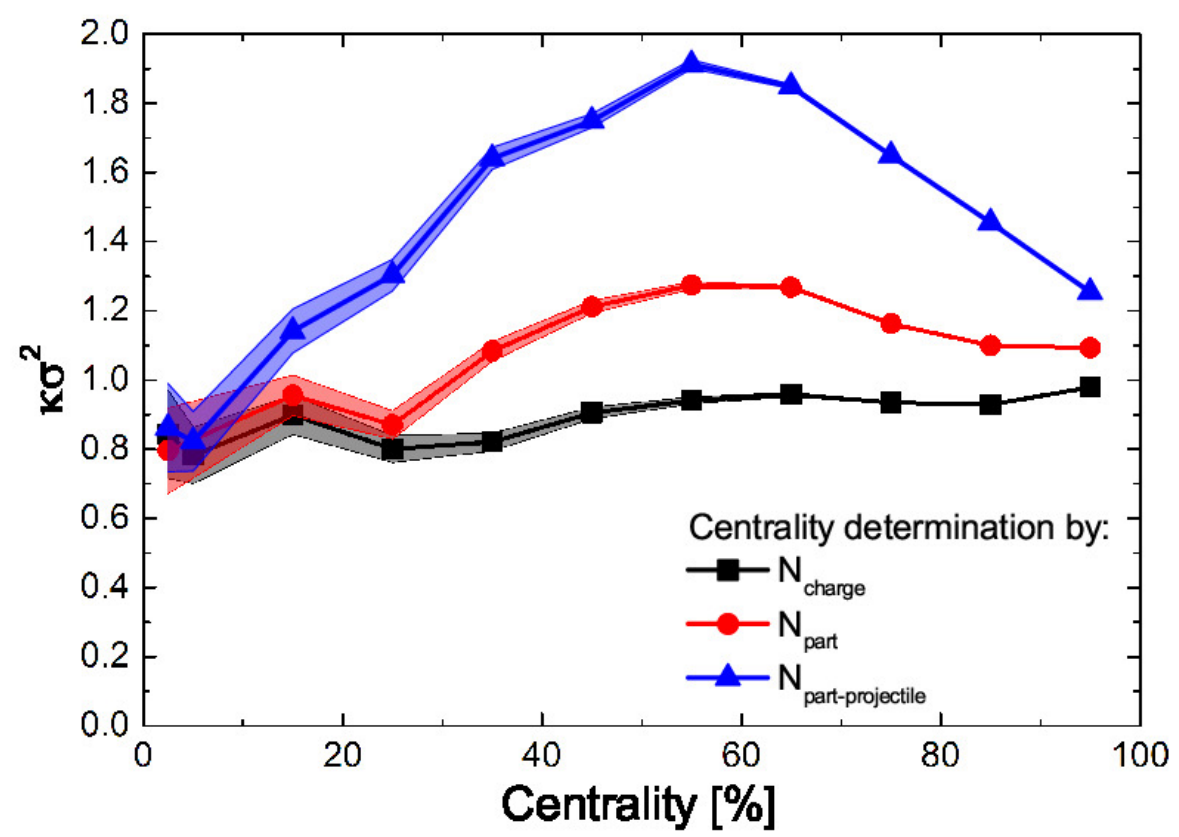

Fig. 2. [Color online] The kurtosis of the net-proton number as a function of centrality which is defined by three different quantities, the number of charged particles, the number of participants and the number of participants in the projectile. Figure from 31.

\subsection{Effect of efficiency}

In practice, particle detectors are not ideal systems but suffer from a finite particle detection efficiency. The efficiency is the number of produced particles that are recorded in the detector divided by the overall yield, see [32]. Therefore, detectors measuring particle number fluctuations will never perform perfectly. We now show how an efficiency below $100 \%$ influences the measurement of cumulants. We begin by studying the effect of efficiency on the fluctuations. In figure 4. we show the result of the net-proton number kurtosis in two different $p_{T}$ ranges as a function of centrality at $100 \%$ and $70 \% N_{\text {charge }}$ efficiency. It is found that the reduced (realistic) efficiency leads to an overall increase of the kurtosis.

Second, we study the effect of a proton efficiency. The result of the variance, skewness and kurtosis of the net-proton number as a function of centrality defined by $70 \% N_{\text {charge }}$ efficiency is shown in figure 5 . We compare the results for $100 \%$ and $75 \%$ proton efficiency. The circles represent calculations with $100 \%$ proton efficiency, the squares are $75 \%$ proton efficiency. We find that the cumulant ratios of the net-proton number for the $75 \%$ proton efficiency is smaller than for the $100 \%$ proton efficiency. 


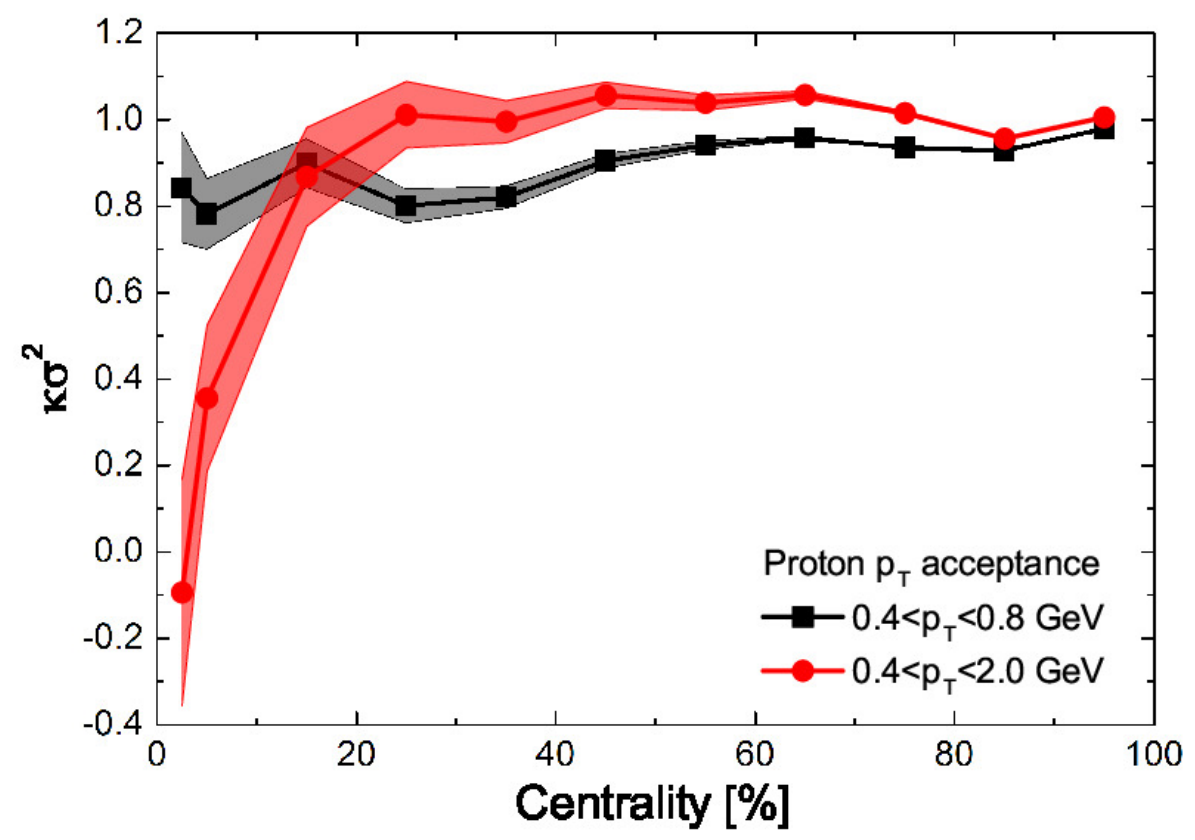

Fig. 3. [Color online] The kurtosis of the net-proton number as a function of centrality (defined by $N_{\text {charge }}$ ) for two different transverse momentum $p_{T}$ ranges. Figure from 31 .

\section{Conclusions}

We have studied the effect of different methods for centrality determination on the measured net-proton kurtosis. We have found that different centrality definitions give different results for the kurtosis. Using a centrality defined by $N_{\text {charge }}$ reduces the effect of volume fluctuations. Moreover, we have studied the effect of two different transverse momentum ranges accepting net-protons in the measurement. We have seen that the wider transverse momentum range leads to a strongly suppressed kurtosis at central collisions. Finally, we have observed the effect of centrality determination, finding a clear impact on the value of the kurtosis.

\section{Acknowledgments}

The computational resources have been provided by the LOEWE Frankfurt Center for Scientific Computing (LOEWE-CSC) and the Center for Computer Services at SUT. This work is supported by the German Academic Exchange Service (DAAD), HIC for FAIR and the Thailand Research Fund (TRF). SS and AL acknowledge support from TRF-RGJ (PHD/0185/2558). CH, AL and YY ac- 


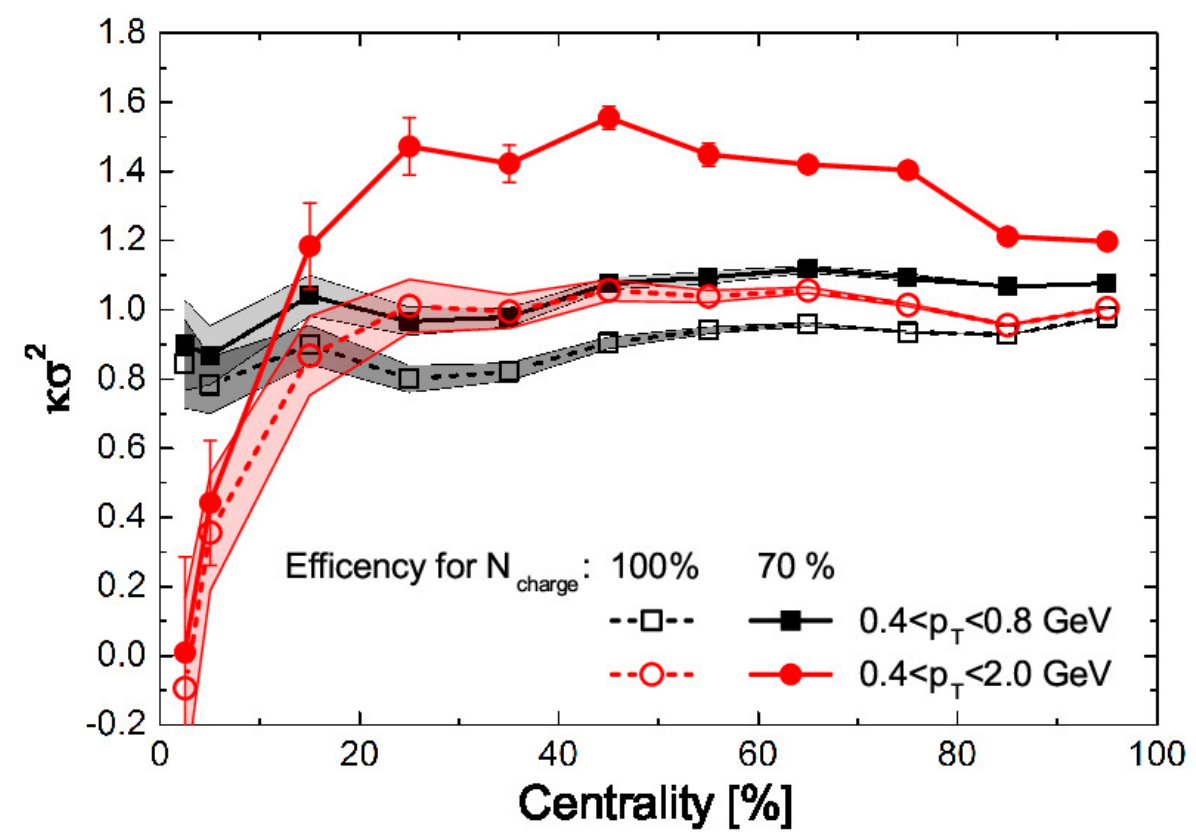

Fig. 4. [Color online] The kurtosis of the net-proton number in two different $p_{T}$ acceptance bins (black squares and red circles) as a function of centrality with $100 \%$ (open symbols) and $70 \%$ (full symbols) $N_{\text {charge }}$ efficiency. Figure from 31.

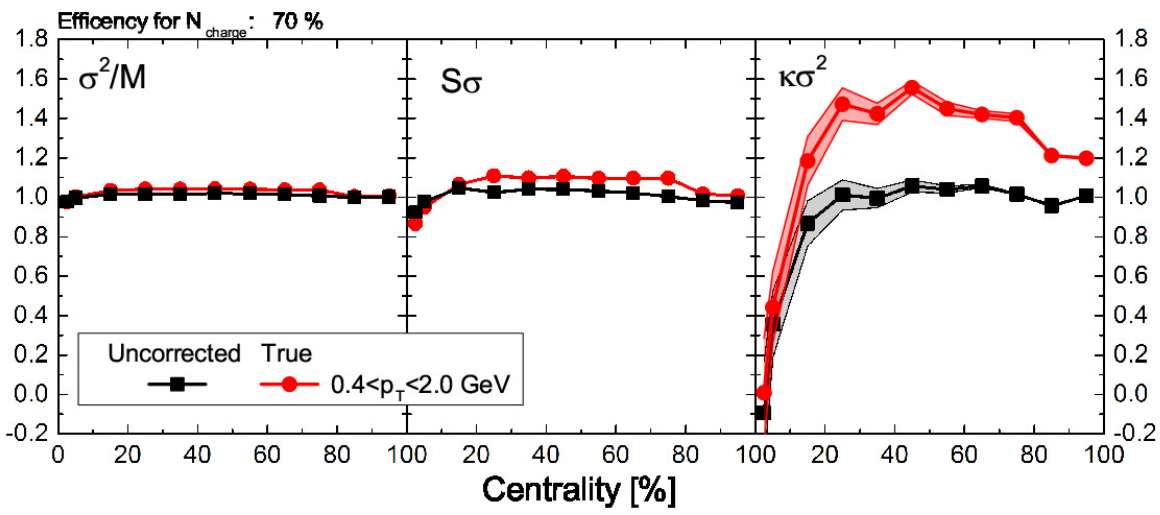

Fig. 5. [Color online] The result of variance, skewness and kurtosis of the net-proton number in two different $p_{T}$ acceptance bins for a $70 \% N_{\text {charge }}$ efficiency. We compare the result between $100 \%$ (red circle symbol) and $75 \%$ (black squares symbol) proton efficiency. Figure from 31. 
knowledge support from Suranaree University of Technology and the Office of the Higher Education Commission under NRU project of Thailand.

\section{References}

1. M. Stephanov, K. Rajagopal, and E. Shuryak, "Signatures of the tricritical point in qcd," Physical Review Letters, vol. 81, no. 22, p. 4816, 1998.

2. J. Randrup, "Spinodal decomposition during the hadronization stage at rhic?," Physical review letters, vol. 92, no. 12, p. 122301, 2004.

3. C. Sasaki, B. Friman, and K. Redlich, "Density fluctuations in the presence of spinodal instabilities," Physical review letters, vol. 99, no. 23, p. 232301, 2007.

4. M. Stephanov, "Non-gaussian fluctuations near the qcd critical point," Physical review letters, vol. 102, no. 3, p. 032301, 2009.

5. C. Herold, M. Bleicher, M. Nahrgang, J. Steinheimer, A. Limphirat, C. Kobdaj, and Y. Yan, "Broadening of the chiral critical region in a hydrodynamically expanding medium," The European Physical Journal A, vol. 54, no. 2, p. 19, 2018.

6. M. Nahrgang, C. Herold, and M. Bleicher, "Influence of an inhomogeneous and expanding medium on signals of the qcd phase transition," Nuclear Physics A, vol. 904, pp. 899c-902c, 2013.

7. M. Szymański, M. Bluhm, K. Redlich, and C. Sasaki, "Net-proton number fluctuations in the presence of the qcd critical point," Journal of Physics G: Nuclear and Particle Physics, vol. 47, no. 4, p. 045102, 2020.

8. M. Cheng, P. Hegde, C. Jung, F. Karsch, O. Kaczmarek, E. Laermann, R. Mawhinney, C. Miao, P. Petreczky, C. Schmidt, et al., "Baryon number, strangeness, and electric charge fluctuations in qcd at high temperature," Physical Review D, vol. 79, no. 7, p. 074505, 2009.

9. W.-j. Fu, Y.-x. Liu, and Y.-L. Wu, "Fluctuations and correlations of conserved charges in qcd at finite temperature with effective models," Physical Review D, vol. 81, no. 1, p. 014028, 2010.

10. L. Chen, X. Pan, F. Xiong, L. Li, N. Li, Z. Li, G. Wang, and Y. Wu, "Statistical and dynamical fluctuations in the ratios of higher net-proton cumulants in relativistic heavy-ion collisions," Journal of Physics G: Nuclear and Particle Physics, vol. 38, no. 11, p. $115004,2011$.

11. F. Karsch and K. Redlich, "Has t c been measured by heavy ion experiments?," Physical Review D, vol. 84, no. 5, p. 051504, 2011.

12. B.-J. Schaefer and M. Wagner, "Qcd critical region and higher moments for threeflavor models," Physical Review D, vol. 85, no. 3, p. 034027, 2012.

13. X. Wang and C. Yang, "Energy and centrality dependence of higher order moments of net-proton distributions in relativistic heavy ion collisions," Physical Review C, vol. 85, no. 4, p. 044905, 2012.

14. D.-M. Zhou, A. Limphirat, Y.-l. Yan, C. Yun, Y.-p. Yan, X. Cai, L. P. Csernai, and B.-H. Sa, "Higher-moment singularities explored by net-proton nonstatistical fluctuations," Physical Review C, vol. 85, no. 6, p. 064916, 2012.

15. P. Rau, J. Steinheimer, S. Schramm, and H. Stöcker, "Conserved charge fluctuations in a chiral hadronic model including hadrons and quarks," Physics Letters $B$, vol. 733, pp. 176-182, 2014.

16. W. Fan, X. Luo, and H. Zong, "Probing the qcd phase structure with higher order baryon number susceptibilities within the njl model," Chinese Physics C, vol. 43, no. 3, p. 033103, 2019. 
17. M. Aggarwal, Z. Ahammed, A. Alakhverdyants, I. Alekseev, J. Alford, B. Anderson, D. Arkhipkin, G. Averichev, J. Balewski, L. Barnby, et al., "Higher moments of net proton multiplicity distributions at rhic," Physical review letters, vol. 105, no. 2, p. 022302, 2010.

18. X. Luo, S. Collaboration, et al., "Probing the qcd critical point with higher moments of net-proton multiplicity distributions," in Journal of Physics: Conference Series, vol. 316, p. 012003, IOP Publishing, 2011.

19. L. Adamczyk, J. Adkins, G. Agakishiev, M. Aggarwal, Z. Ahammed, I. Alekseev, J. Alford, C. Anson, A. Aparin, D. Arkhipkin, et al., "Energy dependence of moments of net-proton multiplicity distributions at rhic," Physical review letters, vol. 112, no. 3, p. 032302, 2014.

20. L. Adamczyk, J. Adkins, G. Agakishiev, M. Aggarwal, Z. Ahammed, I. Alekseev, J. Alford, C. Anson, A. Aparin, D. Arkhipkin, et al., "Beam energy dependence of moments of the net-charge multiplicity distributions in au+ au collisions at rhic," Physical review letters, vol. 113, no. 9, p. 092301, 2014.

21. A. Adare, S. Afanasiev, C. Aidala, N. Ajitanand, Y. Akiba, R. Akimoto, H. AlBataineh, J. Alexander, H. Al-Ta'ani, A. Angerami, et al., "Measurement of higher cumulants of net-charge multiplicity distributions in au+ au collisions at $\mathrm{s} \mathrm{n} \mathrm{n}=$ 7.7-200 gev," Physical Review C, vol. 93, no. 1, p. 011901, 2016.

22. J. Xu, "Energy dependence of moments of net-proton, net-kaon, and net-charge multiplicity distributions at star," in Journal of Physics: Conference Series, vol. 736, p. 012002, IOP Publishing, 2016.

23. B. Abelev, J. Adam, D. Adamová, A. Adare, M. Aggarwal, G. A. Rinella, A. Agocs, A. Agostinelli, S. A. Salazar, Z. Ahammed, et al., "Net-charge fluctuations in pb-pb collisions at s nn= 2.76 tev," Physical Review Letters, vol. 110, no. 15, p. 152301, 2013.

24. A. Rustamov, A. Collaboration, et al., "Net-baryon fluctuations measured with alice at the cern lhc," Nuclear Physics A, vol. 967, pp. 453-456, 2017.

25. A. Ohlson, "Measurements of the fluctuations of identified particles in alice at the lhc," arXiv preprint arXiv:1901.00709, 2019.

26. S. A. Bass, M. Belkacem, M. Bleicher, M. Brandstetter, L. Bravina, C. Ernst, L. Gerland, M. Hofmann, S. Hofmann, J. Konopka, et al., "Microscopic models for ultrarelativistic heavy ion collisions," Progress in Particle and Nuclear Physics, vol. 41, pp. 255-369, 1998.

27. M. Bleicher, E. Zabrodin, C. Spieles, S. A. Bass, C. Ernst, S. Soff, L. Bravina, M. Belkacem, H. Weber, H. Stöcker, et al., "Relativistic hadron-hadron collisions in the ultra-relativistic quantum molecular dynamics model," Journal of Physics G: Nuclear and Particle Physics, vol. 25, no. 9, p. 1859, 1999.

28. G. Graef, J. Steinheimer, F. Li, and M. Bleicher, "Deep sub-threshold $\xi$ and $\lambda$ production in nuclear collisions with the urqmo transport model," Physical Review $C$, vol. 90, no. 6, p. 064909, 2014.

29. C. Patrignani, D. Weinberg, C. Woody, R. Chivukula, O. Buchmueller, Y. V. Kuyanov, E. Blucher, S. Willocq, A. Höcker, C. Lippmann, et al., "Review of particle physics," Chin. Phys., vol. 40, p. 100001, 2016.

30. X. Luo, "Error estimation for moment analysis in heavy-ion collision experiment," Journal of Physics G: Nuclear and Particle Physics, vol. 39, no. 2, p. 025008, 2012.

31. S. Sombun, J. Steinheimer, C. Herold, A. Limphirat, Y. Yan, and M. Bleicher, "Higher order net-proton number cumulants dependence on the centrality definition and other spurious effects," Journal of Physics G: Nuclear and Particle Physics, vol. 45, no. 2, p. 025101, 2017. 
32. B. Abelev, M. Aggarwal, Z. Ahammed, B. Anderson, D. Arkhipkin, G. Averichev, Y. Bai, J. Balewski, O. Barannikova, L. Barnby, et al., "Systematic measurements of identified particle spectra in pp, $\mathrm{d}+\mathrm{au}$, and au+ au collisions at the star detector," Physical Review C, vol. 79, no. 3, p. 034909, 2009. 